\title{
Autour des Equations de Maxwell
}

\author{
Didier Robbes ${ }^{1}$, Thierry Salley ${ }^{1}$, Fréderic Dijoux ${ }^{1}$, Basile Dufay ${ }^{2}$, Gilles Allègre ${ }^{2}$, \\ Maria Timofeeva ${ }^{1}$, Sebastien Morteau ${ }^{1,}$ \\ didier.robbes@inphynix.com \\ Adresse : ${ }^{1}$ Inphynix, Plug N'Work, Bâtiment D, 2 rue jean Perrin, 14460, COLOMBELLES \\ ${ }^{2}$ GREYC - UMR 6072, Université de Caen et ENSICAEN, 6 Bd Mal Juin, F-14050 CAEN Cedex
}

\begin{abstract}
RESUME : Une série de manipulations autour des équations de Maxwell est présentée. Ces travaux s'adressent aux domaines de l'électromagnétisme et de l'algèbre vectorielle dans l'enseignement de la Physique et des Sciences pour l'Ingénieur des licences sciences et technologies, classes préparatoires, écoles d'ingénieur. La problématique générale est posée, en lien étroit avec les matériels utilisés : sources de potentiel, de champ électrique et de champ magnétique, capteurs adaptés, magnétorésistance et bobines de capture, sonde de mesure gradiométrique de potentiel et de champ électrique sans contact. Une quinzaine de manipulations de travaux pratiques sont décrites à des degrés divers, attachées aux 4 équations de Maxwell. La démarche a été mise en œuvre auprès d'étudiants de niveau L2, au travers de la moitié des manipulations décrites. L'ensemble « autour des équations de Maxwell » implique une démarche distribuée au long des cursus, avec des ressorts pédagogiques liés notamment à la récurrence de l'utilisation de concepts, outils numériques, instruments scientifiques et à leurs approfondissements successifs. Elle est ouverte sur des suites possibles à donner : pistes de recherches notamment en didactique des Sciences, et pistes de développements industriels.
\end{abstract}

Mots clés : Equations de Maxwell, Magnétomètre, Electromètre, didactique des sciences, avatar numérique.

\section{INTRODUCTION}

Les équations de Maxwell, piliers de la physique et de nombreux développements technologiques modernes, sont incontournables dans les cursus scientifiques de niveau L. A l'unicité de leur écriture ne correspond pas, pour autant, de protocoles expérimentaux unifiés, facilement utilisables en travaux pratiques. Or, la difficulté de l'appropriation intellectuelle des concepts utilisés dans la formulation de ces équations est reconnue. Nous défendons qu'une série de travaux pratiques dédiés à ces équations est un outil précieux pour améliorer cette phase d'apprentissage intervenant dans une grande partie des niveaux $\mathrm{L}$ des licences de sciences et technologies, ainsi que dans les classes préparatoires aux grandes écoles, les écoles d'ingénieur, notamment avec prépas intégrées, et, dans une moindre mesure, les IUT et quelques sections de formation de techniciens. Cet article est une synthèse de notre contribution encore partielle à cette tâche, mais déjà expérimentée en année 2 de la licence SPI à l'Université de Caen Basse Normandie.

L'ensemble des manipulations présentées et discutées ici couvre les 4 équations de base sous leur forme locale, mais leur forme intégrale y est tout aussi bien prise en compte. Les règles de passage entre ces deux formulations, locale et intégrale, sont reliés aux théorèmes fondamentaux de l'algèbre vectorielle, que sont les théorèmes de Stokes et de Green - Ostrogradsky. Dès lors, le nombre de manipulations décrites est suffisamment élevé pour que nous ayons pu bénéficier d'une présentation en 12 pages en 8 parties. La première pose la problématique générale puis la seconde est dédiée aux caractéristiques générales des matériels mis en œuvre. Chaque équation de Maxwell fait alors l'objet d'une partie, successivement : " autour de Maxwell Faraday », « autour de Maxwell - Gauss », « autour de
Maxwell - Ampère », et « autour de Maxwell - Thomson ». Une discussion argumentée des intérêts pédagogiques sous tendus par notre approche, ainsi que des perspectives R\&D termine notre contribution.

\section{PROBLÉMATIQUE DE L'APPROCHE}

Plusieurs difficultés sont à contourner dès lors que l'on souhaite aborder de façon expérimentale unifiée les équations de Maxwell. La première est liée à un déséquilibre de fait entre les fondements théoriques des grandeurs introduites en statique : charges, potentiel et champ électrique d'une part, courants, flux et champ magnétique d'autre part. Du point de vue appropriation intellectuelle des prémices de ces équations, il apparaît souvent que les premières grandeurs manipulées s'ancrent plus aisément que les secondes dans l'esprit de nos étudiants. Notre interprétation en est que ce fait est associé aux opérateurs mathématiques utilisés : ainsi, pour le jeune bachelier, la généralisation de l'opération de dérivation menant à la définition du gradient d'un scalaire est à priori plus aisée à comprendre que celle $\mathrm{du}$ rotationel d'un vecteur. Ce simple fait pourrait amorcer le déséquilibre initial se créant souvent entre les représentations des grandeurs électriques et magnétiques que se construisent les étudiants.

Une autre difficulté essentielle est liée à un déséquilibre flagrant du point de vue expérimental : la mesure quantitative quasi-statique du champ magnétique est aisée à l'aide de nombreux capteurs disponibles commercialement, alors que celle du champ électrique quasi statique se révèle beaucoup plus délicate, surtout si l'on s'adresse aux faibles valeurs du champ, ne nécessitant que des différences de potentiel de quelques dizaines de volts pour les créer. Jusqu'à très récemment [1], il n'existait pas de capteur réellement adapté à des me- 
sures de faible champ électrique quasi-statique. Nous introduisons un dispositif permettant de résoudre cette difficulté expérimentale, et ainsi contribuer à équilibrer les approches expérimentales des phénomènes électriques et magnétiques fondamentaux.

Une troisième difficulté peut être pointée, dès lors que l'on vise une approche expérimentale unifiée autour des équations de Maxwell : il s'agit de passer de façon progressive du domaine quasi statique, à celui de l'approximation des états quasi stationnaires (AEQS), pour le moins, et, idéalement, jusqu'à inclure les phénomènes de propagation en espace libre. L'investigation de phénomènes du régime d'AEQS est rendue possible grâce à la mise en œuvre de capteurs et de sources de champ à relativement large bande, du continu à quelques centaines de $\mathrm{kHz}$. Les conditions à réunir, pour couvrir simultanément les régimes statiques, quasi-statiques et AEQS font l'objet de la partie suivante de cet article. La propagation n'est pas abordée dans ces travaux.

Enfin, une dernière contrainte est à intégrer sur cette problématique dédiée aux équations de Maxwell : il s'agit de prendre le mieux possible en compte les deux formalisations, locales et intégrales, des phénomènes électromagnétiques. En effet, la nécessité de ces deux formulations complémentaires n'est probablement pas bien comprises par les étudiants. Or, la maîtrise de la manipulation des formalismes associés aux équations de Maxwell ne peut s'apprécier complètement qu'au travers de ces 2 formulations. Ainsi, notre approche se devait - elle d'aborder ces 2 aspects [2]. Ces aspects éminemment pédagogiques seront plus particulièrement détaillés lors de la description de l'équation de Maxwell Thomson.

\section{MATÉRIELS UTILISÉS}

\subsection{Contraintes pédagogiques et cahier des charges}

Comme stipulé en introduction, viser une approche expérimentale unifiée autour des équations de Maxwell implique une maîtrise des grandeurs électro-magnétiques variables dans le temps. L'étendue minimale souhaitable du spectre de fréquence des grandeurs est dépendante de plusieurs facteurs dont : l'étendue de mesure des capteurs/conditionneurs, leurs bande passante, slew rate, dynamique et linéarité. Sont également à prendre en compte la petitesse de certaines grandeurs et paramètres intervenant dans les équations de Maxwell, comme le champ électromoteur associé aux phénomènes de l'induction (voir au 4.1.1), ou encore le produit $\mu_{0} \varepsilon_{0}=10^{-17}$ pondérant le terme de courant de déplacement de l'équation de Maxwell - Ampère. Enfin, la complexité du conditionnement électronique doit être suffisamment faible pour avoir aussi des intérêts pédagogiques dans les niveaux L.

Compte tenu des remarques précédentes, le spectre des signaux utilisés couvre plus de 6 décades de fréquence, jusqu'à une valeur $f_{\max }<1 \mathrm{MHz}$, compatible avec des électroniques à bas bruit, mais d'architectures les plus proches possibles de montages fondamentaux de l'électronique. Ce choix permet d'envisager des réalisations éclatées des conditionnements électroniques actuellement mis en œuvre dans les versions industrielles à composants de surface CMS. Le détail des possibilités offertes ne fait pas l'objet du développement de cet article, indiquons néanmoins que les problématiques fondamentales de l'électronique analogique y seraient abordées : l'amplification de tension et de transimpédance, le traitement différentiel, la contre réaction, la mise à la garde, etc. Nos propositions $[3,4]$ en donnent un large aperçu.

\subsection{Sources des champs électriques et magnétiques}

\subsubsection{Sources magnétiques large bande}

Pour les sources magnétiques, les conséquences des contraintes et choix ci-dessus permettent de dimensionner aisément les circuits à enroulements filaires à mettre en œuvre. Leur première résonance doit intervenir suffisamment au delà de $100 \mathrm{kHz}$, elle en limite le nombre de tours. L'attaque des enroulement doit être le plus simple possible, compatible avec les générateurs de tension basse fréquence sous $50 \Omega$. La connaissance de l'inductance $L$ du circuit, permet alors de décider de l'éventuelle résistance de source $R$ à placer en série sur le circuit pour que la constante de temps $L / R$ soit compatible avec la valeur maximale du spectre des signaux à manipuler. Le choix de $R$ détermine alors le courant maximal délivré par le générateur, et le champ magnétique généré est déductible à partir de la géométrie de l'enroulement considéré. Afin de minimiser les conséquences de l'effet de peau, le choix des fils doit être celui des fils de Litz.

En pratique, le rejet en haute fréquence de la résonance propre de la bobine limite assez vite le nombre de tours, et la limitation du courant d'attaque vers 10 - 100 $\mathrm{mA}$ amène à des valeurs extrêmes des inductions générées de l'ordre de quelques dizaines de $\mu \mathrm{T}$. Ces assez faibles valeurs permettent que les géométries utilisées dans nos expériences se rapprochent de configurations idéales introduites en cours/TD, renforçant ainsi les ponts entre les séquences d'enseignement. Il s'agit essentiellement de boucles circulaires planes, mono et multi tours, ou de boucles carrées. Pour la génération de champ spatialement assez homogène, la configuration de Helmoltz est utilisée. Les dimensions typiques des bobines vont de quelques $\mathrm{cm}$ à quelques dizaines de $\mathrm{cm}$ de diamètre ou de coté. Une conséquence intéressante de la définition des charges au 3.1 est que l'étendue de la plage de fréquences, qui implique une valeur faible du champ $\vec{B}$, entraine aussi une réduction des coûts : moins de masse employée, courants plus faibles, absence de pièces polaires, etc !

\subsubsection{Sources de potentiel et de champ électrique.}


D'un point de vue fonctionnel, ces sources doivent contrôler la valeur du potentiel électrique dans une région spécifiée de l'espace libre, déterminant celle du champ électrique (partie dérivant d'un potentiel). Ces sources sont obtenues à partir de volumes métalliques très conducteurs (Cuivre, Aluminium), de géométrie possédant un haut degré de symétrie (symétrie de révolution). Afin de maîtriser au mieux les inévitables «effets de bords », l'utilisation de propriétés de symétrie géométrique et/ou d'anti-symétrie des distributions de charges des volumes conducteurs par rapport à un plan permet d'imposer un potentiel de référence dans une portion immatérielle de plan, accessible au capteur sans contact. Remarquons que ce choix diffère fondamentalement de la pratique traditionnelle dans laquelle le plan de référence est matériel, conducteur. Au final, symétrie de révolution avec propriétés de symétrie/antisymétrie par rapport à un plan transverse à l'axe de révolution permettent d'obtenir des distributions spatiales de potentiel calculables de façon analytique et/ou numérique, notamment par la méthode des éléments finis réduite à $2 \mathrm{D}$, aisée de mise en œuvre. La figure 1 donne un aperçu d'une source utilisée.

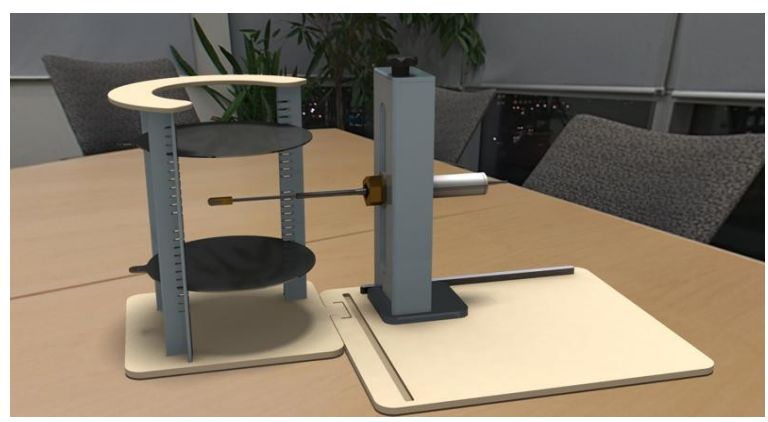

Fig. 1: Vue réaliste de la source de potentiel et de champ en phase finale de développement. Les dispositifs finaux, en cours de réalisation industrielle, sont disponibles depuis fin janvier 2010.

En pratique, une paire d'électrodes métalliques circulaires planes, parallèles et coaxiales (condensateur plan géant) et une sphère métallique creuse sont utilisées, reliées à un générateur BF qui impose la valeur du potentiel de la surface des conducteurs relativement au potentiel de référence. Un montage inverseur à amplificateur opérationnel réalise l'inversion de phase de la source de signal lorsque les distributions de charge souhaitées sur les paires de volumes conducteurs en positions symétriques doivent vérifier une propriété d'antisymétrie. Si la charge d'un des volumes conducteurs doit être mesurée, une capacité étalon de valeur très supérieure à la capacité propre du volume conducteur considéré est intercalée en série. La mesure de la différence de potentiel entre ses bornes permet alors de remonter à la charge recherchée. Ses supports, actuellement en phase finale d'industrialisation, sont disponibles depuis janvier 2010. Les résultats présentés ci dessous ont été acquis sur des prototypes.

\subsection{Capteurs et conditionneurs}

Nous ne donnons ici que les spécifications des capteurs/conditionneurs utilisés, leur conception a fait l'objet de développements particuliers [1], non sans intérêts pédagogiques. Ces derniers sont discutés dans les contributions [3][4] acceptées à cette conférence.

\subsubsection{Induction et flux magnétiques}

La sonde M développée par Inphynix est mise en œuvre. Elle est basée sur une paire de ponts magnétorésistifs à base de magnétorésistances anisotropes de type HMC1022 (Honeywell). Le transfert nominal en sortie du conditionneur est de $20 \mathrm{mV} / \mu \mathrm{T}$, dans $200 \mathrm{kHz}$ de bande passante. Avec une étendue de mesure qui est de $+/-600 \mu \mathrm{T}$, la densité spectrale de fluctuations équi-

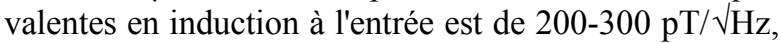
au dessus de quelques dizaines de Hz. Dans $200 \mathrm{kHz}$ de bande passante, la valeur rms de bruit équivalent en champ d'entrée est de $0,7 \mu \mathrm{T}_{\text {rms. }}$. Le procédé de mise en œuvre est abordé en $[3,5]$.

Des mesures de flux magnétiques sont par ailleurs effectuées au moyen de bobines de capture élémentaires, réalisées par simples bobinage de fils de Litz, mono ou multi tours. Lues en tension ou en courant avec des amplificateurs large bande à bas bruit adaptés, la mesure de la force électromotrice induite donne accès à la dérivée du flux, alors que la mesure du courant de court circuit donne une estimation directe du flux magnétique au travers de ces boucles conductrices élémentaires, y compris mono tour, de dimensions centimétriques. Remarquons que cette possibilité de mesure de courants de court circuit pour des boucles de très, très faible impédance diffère des pratiques traditionnelles utilisant uniquement des mesures de tension en circuit ouvert. Ces aspects, en relation avec des travaux de recherche, sont approfondis en [4].

\subsubsection{Potentiel et champ électrique}

La nouvelle sonde sans contact $\mathbf{E}$, développée par Inphynix est utilisée, permettant des mesures sans contact de variations de potentiel électrique et d'une composante de son gradient, projetée le long d'un axe spécifié. Ce capteur gradiométrique, grâce à la mise en œuvre d'asservissements mixte du mode différentiel et du mode commun, a deux sorties simultanées. La valeur de l'écart instantané du potentiel moyen de la sonde par rapport au potentiel de référence du système est disponible en même temps que la différence de potentiel des 2 électrodes symétriques, séparées de la ligne de base $d=5 \mathrm{~mm}$. Cette seconde sortie, gradiométrique, donne donc une mesure du champ électrique projeté sur la ligne de base $d$. Le procédé est détaillé en [5], [6]. Ses intérêts pédagogiques discutés en [3]. Pour le présent article, il suffit de connaître les spécifications du capteur : bande passante $20 \mathrm{mHz}-200 \mathrm{kHz}$, transferts $0,1 \mathrm{~V} / \mathrm{V}$ et $1 \mathrm{mV} / \mathrm{Vm}^{-1}$, étendues de mesures de $100 \mathrm{~V}$ et 


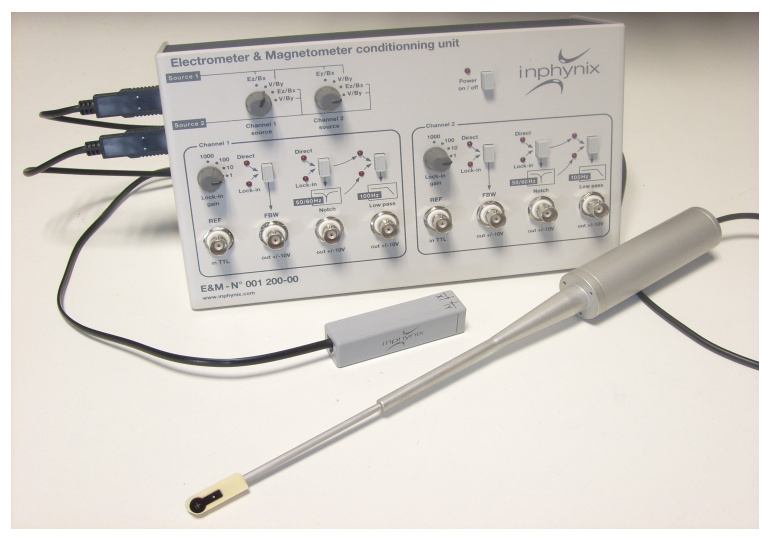

Fig. 2: Sonde E et conditionneur mixte pour la mesure de variations de potentiel et du champ électrique associé. Le dispositif reçoit également les sondes magnétiques. Le conditionneur est doté d'un filtre "notch » à $50 \mathrm{~Hz}$, d'un passe - bas $2^{\text {ème }}$ ordre à $100 \mathrm{~Hz}$ et d'une détection synchrone élémentaire.

de $10 \mathrm{kV} / \mathrm{m}^{-1}$, bruit équivalent en champ à l'entrée de l'ordre de $1 \mathrm{mVm}^{-1} / \sqrt{\mathrm{Hz}}$. Ce nouvel instrument est vu en Fig.2.

\section{EQUATION DE MAXWELL - FARADAY}

\subsection{Forme locale - Forme intégrale}

Une paire de bobines circulaires plates, coaxiales d'axe vertical, de diamètre $20 \mathrm{~cm}$, en position de Helmoltz permet de disposer d'une induction magnétique $\vec{B}$ quasi uniforme, orientée suivant la verticale, sinusoïdale, de fréquence $f$ variable jusqu'à au moins 100 $\mathrm{kHz}$, d'amplitude de quelques $\mu \mathrm{T}$. Un plateau matériel amagnétique, non conducteur, dans le plan médian de la paire permet de positionner magnétomètres et circuits filaires couplés à $\vec{B}$. Dans ces conditions, et c'est un sujet classique de travaux dirigés, il est aisé d'exprimer le champ électrique $\vec{E}$ solution de la première équation de Maxwell :

$$
\overrightarrow{r o t}(\vec{E})=\frac{-d \vec{B}}{d t},
$$

pour obtenir la seule composante non nulle de $\vec{E}$ dans le référentiel du problème, soit $E_{\theta}$ pour le système cylindrique $(r, \theta, z)$ d'origine $\mathrm{O}$ située sur l'axe vertical de symétrie et dans le plan horizontal de symétrie du problème. Il vient pour l'amplitude complexe associable à $E_{\theta}$ :

$$
\bar{E}_{\theta}=-j \omega \bar{B}_{z} r
$$

où $\omega=2 \pi f$, et les barres désignent les grandeurs complexes. Cette expression est valable dans la région où $\vec{B}$ peut être considéré comme spatialement homogène. Une application numérique de (2) borne la valeur maximale accessible au champ électrique, compte tenu des caractéristiques données en 3.2.1, $E_{\theta, \max } \approx 0,05 \mathrm{Vm}^{-1}$ ! En regard des caractéristiques de la sonde de champ disponible données au 3.3.2, force est de constater que la mesure locale directe du champ électromoteur soit plus que délicate : la valeur rms des fluctuations équivalentes en entrée du capteur est de l'ordre de $0,5 \mathrm{Vm}^{-1}$ dans la bande passante du système! Nous reviendrons sur cet aspect au cours de la discussion, mais la conséquence actuelle directe en est que l'approche expérimentale de la forme locale attenante à l'équation (1) est pour le moins très difficile. En revanche, la forme intégrale est beaucoup plus facilement accessible, puisqu'elle aboutit aux lois de l'induction de Faraday et de Lenz. A coté d'expériences traditionnelles à partir de mesures de la force électromotrice induite aux bornes de circuits filaires, généralement multi-tours, de nouvelles expériences ont été réalisées, décrites dans le paragraphe suivant.

\subsection{Nouvelles approches expérimentales}

Cette nouvelle approche est adaptée pour aider l'appropriation de la formule de Stokes, de l'introduction du potentiel vecteur $\vec{A}$ et de ses liens direct avec le champ électromoteur et l'induction. Elle diffère fondamentalement de l'approche traditionnelle des lois de l'induction, fondées sur des mesures de la f.e.m. induite en fonction de la fréquence, du nombre de tours et de l'orientation de circuits filaires. Elle est aussi en cohérence avec les outils numériques, dont les algorithmes résolvent en premier lieu le potentiel vecteur, pour en déduire le champ $\vec{B}$. Dans une première séance de 3 heures, les étudiants se familiarisent avec le problème : géométrie, référentiel, manipulation d'un avatar virtuel 3D de la source de champ au moyen d'un logiciel ergonomique [7] de DAO (voir Fig. 3). Également au cours de cette séance, les étudiants obtiennent de deux façons différentes des évaluations numériques du potentiel vecteur. La première est obtenue par intégration numérique simple, à l'aide du tableur de la suite open office, de l'expression générale de la composante $A_{\varphi}$ en coordonnées sphériques associée à un courant $I$ circulant dans une boucle circulaire de rayon a :

$$
A_{\phi}(r, \theta)=\frac{\mu_{0} I a}{4 \pi} \int_{0}^{2 \pi} \frac{\cos \left(\phi^{\prime}\right)}{\sqrt{r^{2}+a^{2}-2 a r \sin (\theta) \cos \left(\phi^{\prime}\right)}} d \phi^{\prime}
$$

La seconde approche des valeurs de $A_{\varphi}$ est effectuée par les étudiants au moyen du logiciel de simulation numérique par la méthode des éléments finis FEMM, créé par D. Meeker, membre IEEE [8]. A la fin de cette première séance, après avoir comparé les résultats des 2 approches numériques, le replacement de cartographies $2 \mathrm{D}$ issues des simulations numériques au sein de l'ava$\operatorname{tar} 3 \mathrm{D}$ représentant la source de champ, permet à l'étudiant d'affiner la représentation des grandeurs vectorielles manipulées dans le système réel, manipulé dans la séance de TP suivante. Fig. 3 permet d'avoir une pre- 
mière idée du rendu de ce genre de procédé, assimilable conceptuellement à celui dit de «réalité augmentée »en vidéo numérique.
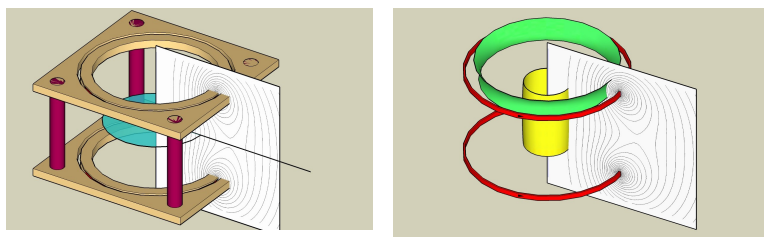

Fig. 3 : à gauche avatar $3 D$ de la paire de bobines en position de Helmoltz, avec importation d'une carte $2 D$ des

lignes de $\left(r A_{\varphi}\right)$. A droite, la maquette est déshabillée, et des surfaces $3 D$, lieux d'isovaleurs de $A_{\varphi}$ sont visualisées.

En seconde séance de TP, les étudiants abordent l'aspect expérimental, en lien direct avec les travaux précédents : puisque seule la composante ortho-radiale de $\vec{A}$ est non nulle, il en va de même du champ électromoteur qui lui est associé, $\vec{E}=-d \vec{A} / d t$. Le passage de la forme locale de la première équation de Maxwell (1) à sa forme intégrale s'effectuant selon le théorème de Stokes, il est alors possible de construire des contours d'intégration donnant accès à des circulations de $\vec{A}$ particulièrement simples à exprimer dès lors que les contours choisis sont une réunion de segments parallèles ou perpendiculaires au vecteur unitaire $\vec{u}_{\phi}$. De tels contours sont simplement : soit des secteurs circulaires centrés sur l'axe de symétrie du problème, soit des contours rectangulaires dont la normale au plan contenant le contour est radiale. Lorsque de tels contours sont matérialisés au moyen de bon conducteurs filaires, la loi de Lenz en configuration circuit ouvert, et/ou la configuration de court circuit permettent de remonter au flux au travers du contour. Des évaluations du champ électromoteur puis du potentiel vecteur, sont alors déductibles des mesures de tension ou de courant de court circuit, à comparer aux évaluations numériques de la première séance. Fig. 4 montre des dessins de tels circuits, dont les réalisations pratiques concrètes ont été mises en œuvre par les étudiants. Le choix des circuits matérialisant ces contours permet d'obtenir plusieurs résultats expérimentaux. Une série de secteurs de cercles de rayon en progression arithmétique permet d'obtenir $A_{\varphi}(r, \pi / 2)$ à comparer à ce que peut donner la simulation sous FEMM, ou à partir de l'expression (3). Des circuits rectangulaires, de longueur 1 plutôt en progression géométrique, permettent d'obtenir la valeur quasi locale de $A_{\varphi}(r, \pi / 2)$ lorsque 1 est largement supérieur au rayon des bobines. En effet, dans ces conditions, seule la circulation de $\vec{A}$ le long du petit coté du circuit qui est dans le plan horizontal de symétrie du système est significative. Le petit coté de ces circuits rectangulaires a été choisi se superposant à l'un des arcs des secteurs de cercle utilisés dans la manipulation précédente, l'étudiant doit discuter du lien et de la cohérence entre les 2 types de mesures.
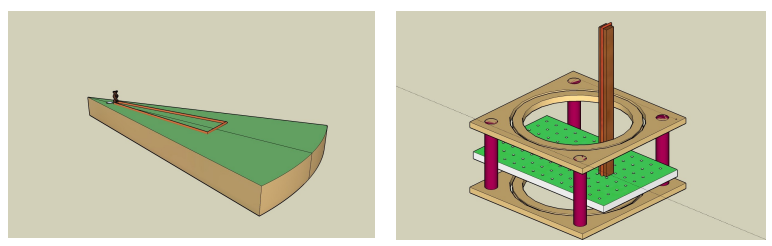

Fig. 4 : à gauche dessin d'un circuit en forme de secteur circulaire. La circulation de $\vec{A}$ le long des 2 rayons est nulle, celle le long de la portion de périmètre est obtenue de façon élémentaire. A droite, dessin d'un contour rectangulaire allongé. La circulation de $\vec{A}$ le long des brins verticaux est nulle. Le long des petits brins horizontaux, les contributions s'opposent, mais le poids des contributions est différent, suivant la longueur du contour rectangulaire.

\section{EQUATION DE MAXWELL - GAUSS}

\subsection{Contraintes et difficultés}

\subsubsection{Statique, Quasi-Statique, Quasi-Stationnaire}

Pour cette seconde équation de Maxwell :

$$
\operatorname{Div}(\vec{E})=\frac{\rho}{\epsilon_{0}},
$$

une réalisation concrète locale de l'opération $\operatorname{Div}(\vec{E})$ nécessiterait idéalement le contrôle d'une charge localisée, enfermée dans un petit cube immatériel assimilable à un volume élémentaire, et l'on viendrait y échantillonner la composante normale du champ électrique sur chacune des faces. Telle situation n'est guère facile à réaliser, sauf dans le cas $\rho=0$, mais alors l'intérêt est limité, nous reviendrons sur cette problématique lors de la discussion finale. A nouveau c'est donc la forme intégrale associée à l'équation (4) qui s'avère plus aisée d'accès expérimental. D'un point de vue formel, elle s'obtient par application du théorème de Gauss, nécessitant la définition d'une surface fermée entourant une distribution de charge, dont le flux est donné localement par la composante normale du champ électrique résultant de cette distribution. Le cas académique est celui de la sphère conductrice chargée, isolée. Cette dernière condition ne peut être strictement remplie que dans le cas statique, car la modification de l'état de charge ne peut se faire sans rupture au moins momentanée de l'isolation de la sphère. Par ailleurs, la nature de la détection du champ $\vec{E}$ par la sonde $\mathbf{E}$ disponible mène à une mise en œuvre grandement facilitée en alternatif. Rappelons surtout que la problématique générale posée dans la partie I de ces articles implique d'envisager le régime variable, aussi bien du point de vue des grandeurs électriques que magnétiques. Notons qu'en cela notre approche diffère de l'approche traditionnelle, dans laquelle le régime de l'approximation des états quasi stationnaire s'adresse pour l'essentiel aux grandeurs magnétiques. Prenant en compte ces arguments, il est alors tout à fait légitime d'appréhender l'équation (4) en régime lentement variable. La diver- 
gence de l'éventuelle partie rotationnelle de $\vec{E}$ est par nature nulle et les choix d'expériences sont effectués pour que les effets purement électriques soient très très largement dominants.

\subsubsection{Difficultés de la sphère en régime harmonique}

Il existe une difficulté supplémentaire liée au caractère idéal de la sphère isolée, dont les réalisations pratiques sont difficiles à maîtriser ! A titre d'exemple illustrant notre propos, le cas pratique du lien filaire de la sphère à une source de potentiel a été quantifié par une simulation sous FEMM [8]. La planche Fig. 5 permet de visualiser la difficulté : les amenées des charges, et plus généralement l'environnement proche, peuvent modifier de façon sensible les valeurs prises par le potentiel et le champ électrique environnant la sphère par rapport à celles du cas idéal. Dès lors, le choix des expériences menées doit intégrer la contrainte de la prise en compte de ces paramètres. La conséquence était, jusqu'à des époques assez récentes, alors très pénalisante car les solutions analytiques répondant à des géométries de plus faible symétrie deviennent très rapidement difficiles à formuler explicitement, inadaptées d'ailleurs aux niveaux L1, L2.

\subsubsection{Sources de potentiel et de champ en alternatif}

Le cas académique de la sphère est difficile à maîtriser, et les solutions de l'équation de Poisson permettant de trouver le champ de potentiel scalaire sont rapidement inextricables analytiquement. Toutefois, la révolution numérique permet de contourner cette difficulté. En effet, c'est désormais presque un jeu d'enfant que de résoudre des problèmes d'électrostatique et d'AEQS par la méthode des éléments finis. Dès lors, ce que l'on peut attendre de distributions de charges répondant à une symétrie de révolution devient facile d'accès, y compris pour les niveaux de L1 et de L2. En pratique, les supports d'expériences répondent au cahier des charges décrit au 3.1.2, notamment avec une zone de mesure possédant une symétrie axiale et un plan de symétrie/antisymétrie perpendiculaire à l'axe de révolution $o z$. En particulier, le cas de deux électrodes conductrices planes, circulaires, coaxiales et parallèles est très facile à traiter aussi bien numériquement qu'expérimentalement avec le matériel présenté en Fig. 1 et Fig. 2. Cet arrangement de base est un "condensateur géant», au sein duquel potentiel et champ vont pouvoir être mesurés en fonction des coordonnées d'espace, en régime de charge harmonique et donner lieu aux expériences décrites ci-après.

\subsection{Gradient, Projection, Flux et Divergence}

\subsubsection{Gradient du potentiel, projection du champ}

La première expérience de base, à mener typiquement
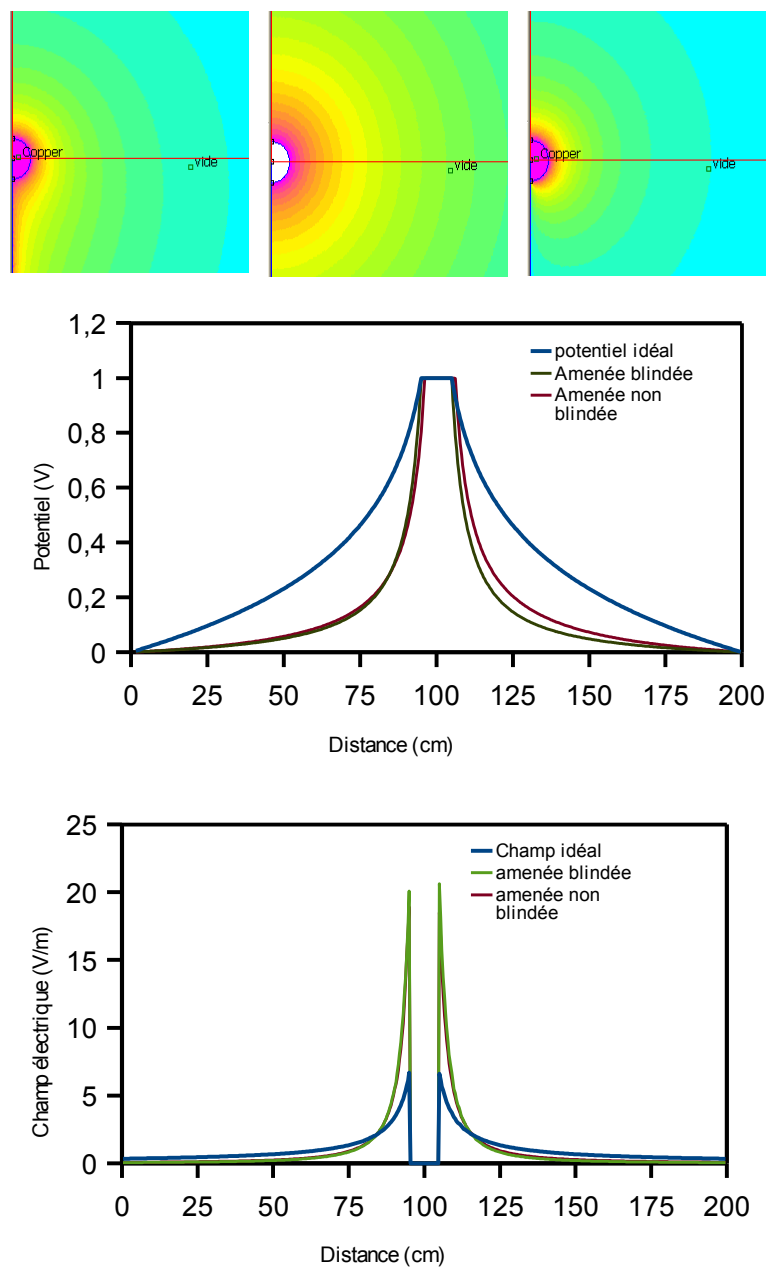

Fig 5 : Ces planches montrent la difficulté d'obtenir une situation expérimentale réalisable (en haut à gauche et à droite) correspondant au cas idéal de la sphère isolée (en haut, au centre).Dans les 3 cas, les sphères sont au potentiel de $1 V$. Les évolutions des tracés de V et de $|\vec{E}|$ sont reportées dans les 2 graphiques ci-dessus, montrant l'effet d'une amenée filaire de longueur $=2 x$ (diamètre sphère).

en $\mathrm{L} 1$, consiste à relever les variations de potentiel $V$ et la composante $E_{z}(z)$ du champ $\vec{E}$ entre les armatures, le long de l'axe de révolution $o z$, permettant de vérifier leurs compatibilité avec la simulation numérique et avec la relation fondamentale $\vec{E}=-\overrightarrow{\operatorname{grad}}(V)$. A un niveau plus averti, en intégrant un minimum le principe de fonctionnement de la sonde $\mathbf{E}$, il est également possible de discuter des effets d'influence entre les électrodes de la sonde, et les parois métalliques de la source.

La seconde expérience, également en L1, consiste à faire le relevé de $V$ et de $\vec{E}$ au centre de l'espace inter armatures, en fonction de l'angle $\theta$ entre le vecteur unitaire de l'axe $o z$ et la direction de référence de la sonde gradiométrique. Cette expérience permet un travail sur la projection pour obtenir la composante $E_{\theta}(0,0)$, parfaitement utile au niveau L1pour nombre d'étudiants! 
Une troisième expérience, toujours en L1, permet de mesurer la composante $E_{z}(r, 0)$ dans le plan de symétrie de l'expérience, que l'on compare aux résultats numériques voir analytiques pour ceux qui en ont le courage, mais les fonctions de Bessel et autres polynômes de Legendre ne sont pas accessibles aux étudiants de L1. Toutefois, la seule vue de l'expression analytique par ces jeunes étudiants qui appréhendent en général pour la première fois un outil de simulation numérique, permet de les sensibiliser à la puissance et la relative facilité de mise en œuvre de ce type d'outils numérique.

\subsubsection{Théorème de Gauss}

Cette expérience est plutôt à effectuer lors de l'énoncé des équations de Maxwell, et de l'invocation de leur compatibilité avec les acquis antérieurs. Elle est donc plutôt à mener typiquement en année 2 des cursus. Elle permet la reprise de l'outil numérique utilisé (récurrence de l'utilisation des outils), avec approfondissements des opérations menées : définition du flux, intégrales doubles, etc. A ce stade de l'apprentissage, les mécanismes de réduction de dimensions de problèmes par symétrie géométriques et physiques ne sont en général pas bien stabilisés dans l'esprit des étudiants. L'introduction de la manipulation d'avatar $3 \mathrm{D}$, telle que présentée en 4.2 Fig. 3, permet une approche ergonomique de la description des opérations à mener pour réussir le TP.

Dans la première phase «mesure » de l'expérience, la composante normale du champ, $E_{n}(r, z)$, est relevée le long d'un trajet défini dans un plan vertical contenant l'axe de symétrie, reliant 2 points de l'axe $o z$. Le trajet est choisi de façon que la surface engendrée par sa révolution autour de $o z$ englobe l'une des électrodes. L'idée est alors de déduire de ces mesures le flux global au travers de la surface, à relier aux charges de l'armature. De façon assez naturelle, le passage de la série de valeurs mesurées $E_{n}(r, z)$ à l'intégrale de surface passe par la notion d'échantillonnage, et comme le lecteur pourra l'apprécier dans la suite, la technique sera employée plusieurs fois au cours des séances successives. Les valeurs des échantillons $E_{n}(r, z)$ sont alors pondérées par les surfaces des couronnes élémentaires définies à partir du pas d'échantillonnage le long du trajet, puis sommées entre elles. Dans cette étape, la manipulation des avatars 3D est assurément un plus pour que l'étudiant arrive à une perception globale de la manipulation, du passage des échantillons à valeurs locale, à une grandeur intégrale. Le résultat global est alors une estimation expérimentale du flux des charges de l'électrode englobée, au travers de la surface engendrée par le trajet. Il s'agit donc bien là du premier volet d'une réalisation du théorème de Gauss. Fig. 6 montre Un résultat de la phase numérique sous FEMM. Une version prototype du support d'expériences Fig. 1 a été utilisée pour collecter les données de la Fig. 7. S'y superposent remarquablement, sans ajustement, les données expéri-mentales et celles issues de la simulation numérique.
Enfin, le graphe Fig. 8 donne un aperçu du rendu de la technique de représentation/médiation cognitive d'expériences au travers d'avatars virtuels.

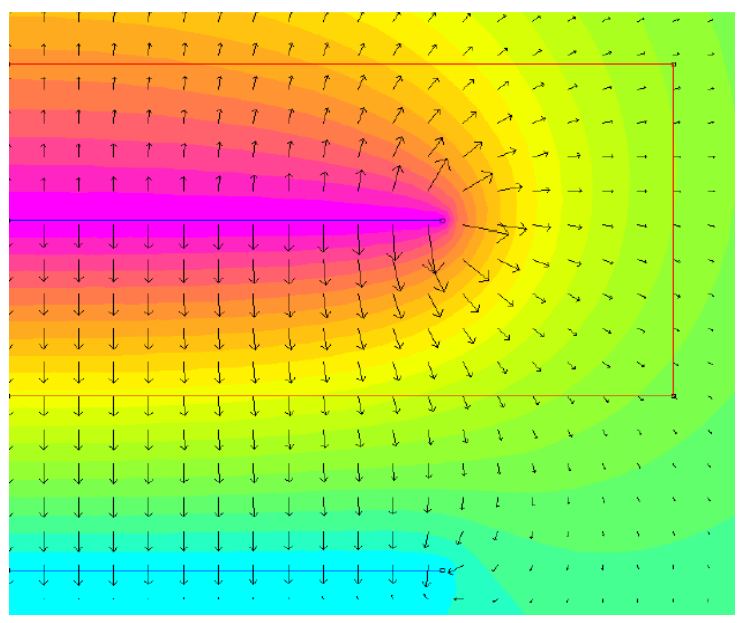

Fig. 6 : Fenêtre de post processing sous FEMM. L'axe de symétrie oz est vertical, à gauche. Les traits bleu sont la trace des électrodes métalliques circulaires de rayon 10 $\mathrm{cm}$. Le trait rouge est la trace du chemin où sont estimés et mesurés les échantillons $E_{n}(r, z)$

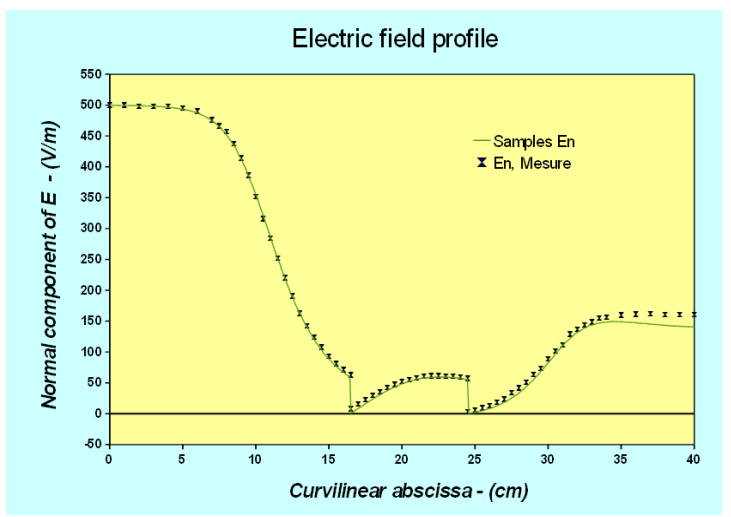

Fig. 7 : Manipulation autour du théorème de Gauss. Les échantillons de la composante normale de $\vec{E}$ calculés sous FEMM se superposent aux données mesurées sur une très grande partie du contour. Le départ du trajet est au centre des armatures.

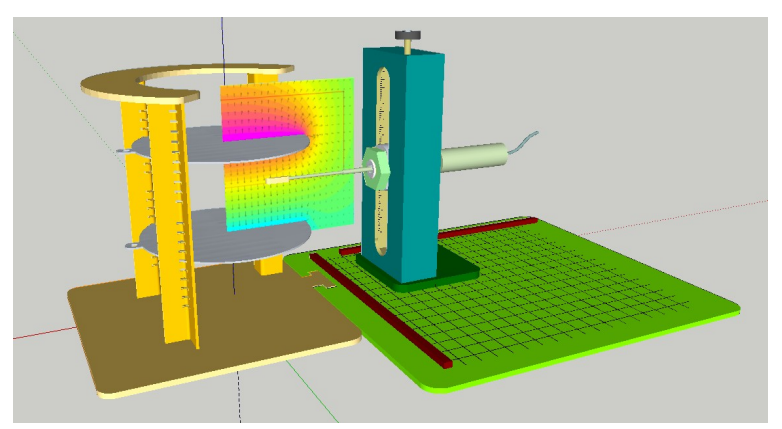

Fig. 8: Utilisation de la technique des avatars numériques d'expériences, pour replacer les données dans leur contexte environnemental. Ce type de représentation/médiation est supposé efficace, du point de vue cognitif. 
Pour aller plus loin dans cette réalisation expérimentale du théorème de Gauss, il est alors nécessaire de mesurer par ailleurs les charges de l'électrode, le rapport du flux estimé et des charges mesurées devant être compatible avec la constante diélectrique du vide $\varepsilon_{0}$. L'ensemble de cette expérience est en cours de finalisation sous sa version industrielle, pour janvier 2010. Les expériences décrites dans ce paragraphe sont donc en bonne voie pour être mises en œuvre par des étudiants.

\section{EQUATION DE MAXWELL - AMPÈRE}

Dans le développement de nos travaux, cette expérience est la plus ancienne, présentée notamment au CETSIS 2003 à Toulouse [9]. Pour cette raison, elle ne sera que peu détaillée ici, nous en donnons les tenants et les aboutissants. La sonde $\mathbf{M}$ est utilisée pour mesurer l'induction magnétique autour d'un circuit filaire parcouru par un courant sinusoïdal $I$. Le circuit est une simple spire carrée, parcourue typiquement par un signal de l'ordre de $100 \mathrm{~mA}$, délivré par un générateur de fonction $(50 \Omega)$ à quelques $100 \mathrm{~Hz}$. Le support d'expérience dédié (Fig. 9) permet de définir des contours de formes et de tailles variées (carré,cercles,...). Le long de ces contours, la composante tangentielle $B_{t}$ de l'induction $\vec{B}$ peut être échantillonnée, au pas de $1 \mathrm{~cm}$. Une fois saisis, les échantillons sont pondérés par le pas d'échantillonnage, puis sommés afin d'obtenir une estimation de la circulation de $\vec{B}$ le long du contour étudié, qui peut entourer ou non le fil parcouru par le courant $I$. La mesure de ce courant $I$ permet alors à l'étudiant de vérifier que le rapport de l'estimation de la circulation au courant $I$ tend bien vers la valeur de la perméabilité magnétique du vide $\mu_{0}$. Dans ces manipulations, le théorème d'Ampère, déduit de forme limitée de la $3^{\text {ème }}$ équation de Maxwell $\operatorname{rot} \vec{B}=\mu_{0} \vec{j}$, est donc abordé, avec la problématique précédente de l'échantillonnage spatial d'une composante du champ, suivie d'une estimation d'une intégrale par sommation discrète sur les échantillons. Il est intéressant de remarquer que le rendu de la valeur de $\mu_{0}$, à $2-3 \%$ près est tout à fait pertinent, et valide complètement la procédure d'échantillonnage et d'évaluation de l'intégrale curviligne associée à l'opération de circulation. Cette décomposition, pas à pas, nous avait semblé, à l'époque, très pertinente pour aider les étudiants. Plusieurs promotions d'entre eux ont pu réaliser ce TP. Pour une bonne efficacité, il est recommandé de prévoir suffisamment de temps, et de ne pas mettre cette expérience en fin de séance.

\section{EQUATION DE MAXWELL - THOMSON}

\subsection{Travaux autour de la forme locale}

\subsubsection{Principe}

Si les expériences décrites précédemment se réfèrent aux formes intégrales associées aux équations de Max

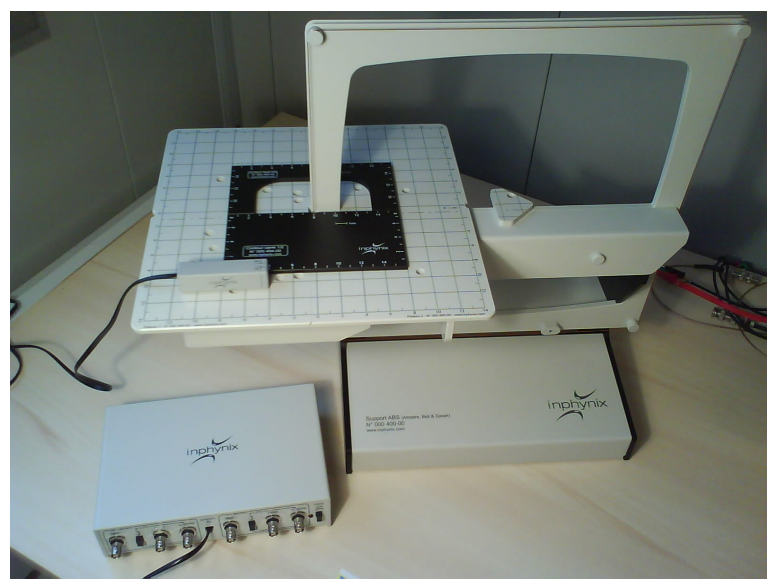

Fig 9 : Support d'expérience ABS (Ampère - Biot -

Savart). Son plateau amovible permet d'étudier la spire carrée, et de positionner des contours de repérage de l'abscisse curviligne le long de contours d'intégration lors de la réalisation de la circulation de $\vec{B}$.

well, la dernière équation : Div $\vec{B}=0$ va donner lieu à des manipulations mieux adaptées à la forme locale. L'opération nous semble d'un grand intérêt pédagogique, car elle permet une réalisation concrète de l'opération «divergence », maintes fois décrite au tableau noir autour d'un petit cube élémentaire où sont attachés, au centre de chaque face, des vecteurs permettant d'écrire cette opération vectorielle, bien appréciée des étudiants, parce que simple à mémoriser. Le bilan des flux élémentaires entrants et sortants en est également déduit. Le principe utilisé ci-après est le suivant : au sein d'une région de champ moyennement in-homogène, obtenue par exemple par une attaque anti symétrique de la paire de bobines en position de Helmoltz, la composante normale $B_{n}$ de $\vec{B}$ est mesurée sur les 6 faces d'un petit cube. Celui ci est assimilable à un volume élémentaire dès lors que la variation spatiale de $\vec{B}$ lorsque l'on déplace le magnétomètre dans le plan de chaque face de ce petit cube reste petite devant la valeur moyenne $\langle\vec{B}\rangle$ sur cette face. Dans ces conditions, il est possible d'obtenir les 6 valeurs de $B_{n}$ nécessaires : d'une part à l'estimation de l'opération de divergence, et d'autre part, au bilan du flux entrant et du flux sortant du cube élémentaire.

\subsubsection{Opérateur « Divergence »}

A partir des 6 valeurs de $B_{n}$, et des dimensions du cube pseudo élémentaire, une estimation de l'opération locale de divergence est obtenue en formant :

$$
\frac{d B_{x}}{d x} \approx \frac{B(x+\Delta x)-B(x)}{\Delta x},
$$

opération répétée sur les 2 autres composantes. La somme des 3 résultats est une estimation de la divergence de $\vec{B}$. La question de trouver 0 ou non est alors une belle source de discussions au sein du groupe d'étu- 
diants en action, car bien entendu, on ne peut trouver strictement zéro, dans l'absolu ! Une réflexion autour de la bonne prise en compte de la marge d'incertitude de la mesure est à mener, afin de juger de la pertinence ou non du résultat. A ce stade du TP, les étudiants peuvent être laissés dans l'expectative, la suite du déroulement des manipulations devant pouvoir leur permettre d'affiner leur première analyse !

\subsubsection{Bilan des flux pseudo élémentaires}

La mesure des 6 composantes de $B_{n}$ sur les 6 faces du cube permet aussi d'obtenir les flux pseudo élémentaires correspondants, au travers de chacune des faces. Le bilan des flux entrants et sortants du cube est alors accessible, la +/- grande déviation du résultat par rapport à zéro vient ainsi alimenter une première fois la discussion engagée au paragraphe précédent.

Pour corser cette approche pseudo locale, et compte tenu des compétences acquises lors des séances de TP relatives à la première équation de Maxwell, les arêtes du cube élémentaire peuvent être matérialisées, en y attachant des circuits filaires carrés épousant chacune des faces ! En refaisant des mesures de fem induite ou de courant de court circuit décrites au 4.2, il est alors possible d'avoir une bonne estimation des flux élémentaires au travers de chaque face, et comparer à nouveau aux résultats précédents. Cette partie utilise le ressort pédagogique de l'appel à un savoir - faire récemment acquis, dans le but notamment de renforcer cette acquisition, faute de quoi elle est, et sera, d'autant plus incertaine si elle n'est pas remise en œuvre suffisamment rapidement. Ceci sera pointé en dernière section de cette article.

\subsubsection{Aboutissement des mesures « forme locale »}

A l'instar du ressort pédagogique précédent, mais cette fois entre Travaux Pratiques et Cours Magistraux (math appliquées, physique), les 3 paragraphes précédents donnent maintenant l'occasion de revenir sur les fondements du théorème de Green - Ostrogradky, à partir de l'équation (2) sommée avec celles attenantes aux 2 autres composantes. Il suffit de multiplier par l'élément de volume, pour que les flux élémentaires entrants et sortants réapparaissent, établissant l'équivalence locale entre le bilan élémentaire des flux et la divergence pondérée par le volume élémentaire. C'est avec cette remarque que l'étudiant peut, d'une part, finir de juger de la pertinence des protocoles de mesure précédents, relativement à la quatrième équation de Maxwell, Div $\vec{B}=0$. D'autre part, le lien au cours magistral, induit les expériences ci après, dédiées à la formulation intégrale de l'équation de Maxwell - Thomson,

\subsection{Forme Intégrale - Théorème de Green Ostrogradsky - Conservativité de B.}

La considération d'une collection de volumes élémentaires accolés et empilés faces à faces, permet d'établir le théorème de Green - Ostrogradsky. Son application aux grandeurs de la quatrième équation de Maxwell permet ainsi d'établir la propriété de conservativité du flux du champ $\vec{B}$. Une dernière expérience permet d'en rendre compte. Décrite initialement brièvement en [1], elle bénéficie désormais d'un support dédié (voir Fig. 10), se présentant sous la forme d'un plateau carré $(32 \times 32 \mathrm{~cm})$, dont le pourtour reçoit un enroulement filaire (24 tours de fil). Un contour carré et un pavage sont sérigraphiés sur le plateau, guidant l'échantillonnage spatial de la composante normale $B_{n}$ sur la surface plane délimitée par le contour, au pas de $2 \times 2 \mathrm{~cm}^{2}$. L'utilisation judicieuse des symétries permet de réduire le nombre d'échantillons à prélever pour couvrir la surface. Au final, la sommation discrète des échantillons, pondérées par les pseudo éléments de surface permet de remonter au flux englobé par le circuit. Afin de vérifier la compatibilité de cette mesure de flux avec la propriété de conservativité, une seconde surface, s'appuyant sur le même contour est échantillonnée, suivant le même protocole. Cette surface est matérialisée par une pyramide amovible sur le plateau carré (Fig. 10). L'étape finale de la manipulation consiste alors à comparer les deux valeurs de flux obtenue, et discuter de la pertinence du résultat, notamment eu égard aux erreurs d'arrondi associables à l'échantillonnage de $B_{n}$ sur la face de la pyramide. Le cas échéant, l'étudiant peut reconsidérer les sommations discrètes effectuées, par des pondérations simples des échantillons prélevés sur les bords, de forme trapézoïdale ou triangulaire, ce qui revient à adapter le pas d'échantillonnage. En pratique, lorsque l'ensemble de la manipulation est bien mené, l'accord entre les deux estimations de flux est excellent. Le ressort pédagogique ici est à nouveau l'emploi récurrent de la technique d'échantillonnage, avec approfondissements successifs. Il est en effet raisonnable de considérer que l'aptitude des étudiants à discuter de la pertinence des résultats ne puisse intervenir qu'à partir d'une connaissance minimale des outils mis en œuvre, et il est peu probable que ce processus puisse se dérouler sur la durée d'une seule séance. Nous pensons que le chaînage de plusieurs séances autour d'un thème central permet d'aborder mieux le difficile apprentissage de la rigueur scientifique.

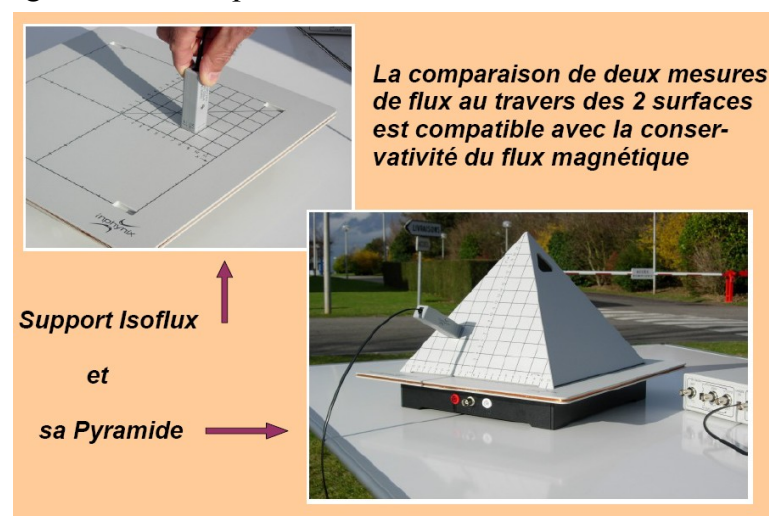

Fig. 10 : Support dédié au théorème de Green - Ostrogradsky et à la conservativité du champ $\vec{B}$. 


\section{DISCUSSION - PERSPECTIVES}

\subsection{Bilan}

Nous venons de présenter la problématique générale sous tendant notre approche pédagogique : un thème fédérateur, celui des équations de Maxwell, permet la mise en œuvre de concepts mathématiques (algèbre vectorielle), d'outils numériques (tableurs, méthode des éléments finis,...), d'instruments scientifiques (capteurs, conditionneurs de signaux, système d'acquisition,...), tout en s'appuyant très fortement sur l'approche expérimentale. Une quinzaine d'expériences sont décrites dans ces 7 parties. Une bonne moitié de celles - ci ont été testées par les étudiants de L2 de la licence Sciences Pour l'Ingénieur de l'Université de Caen Basse Normandie, au printemps 2009. Quelques unes sont utilisées depuis les années 2003. Ces expériences sont en très grande partie totalement nouvelles :

- du point de vue des contenus : travaux autour de champs peu utilisés (potentiel vecteur, champ électromoteur, potentiel scalaire et champ électrique quasi-statique de l'espace libre), travaux autour des opérateurs de l'algèbre vectorielle (projection, gradient, circulation, flux, divergence), travaux autour de la complémentarité des formulations locales et intégrales, travaux autour de configurations académiques en $\mathrm{CM}$ et TD.

- du point de vue des instruments : capteurs et conditionneurs large bande, faible bruit et grande dynamique, amplification de transimpédance adaptée à de très faibles impédances, et surtout mise en œuvre d'un volmètre - gradientmètre sans contact à haute sensibilité.

- du point de vue de la méthodologie : utilisation d'outils de visualisation 3D, utilisation d'avatars virtuels correspondant aux maquettes réelles, permettant démontage, intégration de données 2D, réalité augmentée, utilisation récurrente et progressive d'outils numériques disponibles en ligne gratuitement, recherche de l'équilibre et de l'unicité entre les mesures électriques et magnétiques, recherche de liens entre séances successives, $\mathrm{CM} / \mathrm{TD}$, et surtout avec de l'interdisciplinarité Mathématiques appliquées - Physique - Sciences Pour l'Ingénieur.

\subsection{Lacunes}

Comme déclaré en introduction générale, cette contribution est encore partielle. Pointons ici les compléments qu'elle mériterait. Plusieurs aspects des équations de Maxwell ne sont pas abordés :, notamment courants de déplacement $\mu_{0} \epsilon_{0}(d \vec{E} / d t)$ (équation de Maxwell Ampère) et propagation. Il y a un déséquilibre trop important entre les travaux dédiés à la forme locale et ceux dédiés à la forme intégrale : seule la dernière équation de Maxwell fait l'objet d'un protocole de ré- flexion approfondi du passage d'une forme à l'autre, autour de l'opération de divergence et du théorème de Green - Otrogradky. Il serait pertinent d'en avoir le pendant pour $\overrightarrow{\operatorname{rot}}(\vec{E}), \overrightarrow{\operatorname{rot}}(\vec{B})$ et pour le théorème de Stokes, d'une part, et concernant Div $\vec{E}$, d'autre part.

\subsection{Perspectives}

\subsubsection{Autour du thème des équations de Maxwell}

Les lacunes mentionnées ci-dessus donnent ipso-facto des pistes de réflexions, recherches et développements envisageables. Concernant nos propres développements, une thèse est en cours sur le magnétomètre dual de notre sonde E. Les travaux attenants à cette thèse ont permis l'approche de mesure de flux par mesure de courant de court circuit décrits en 4 , mais surtout ils visent une dynamique accrue de capteurs magnétiques, de type bobine de capture miniature, à partir de la fraction de $\mathrm{pT} / \sqrt{ } \mathrm{Hz}$. Le lecteur averti conçoit dès lors que notre remarque énoncée au paragraphe $\mathbf{3 . 1}$ sur la conséquence de la petitesse de $\mu_{0} \epsilon_{0}$ ne soit pas forcément rédhibitoire ! Notre objectif concret est en effet d'être en état de mesurer, au sein d'une structure de type « condensateur géant » analogue à celle de la Fig. 1, le champ magnétique associé au terme de déplacement seul. Du coup, si nous devenons capables de mener cette expérience, dans une gamme de fréquence de 100 $\mathrm{Hz}$ à $100 \mathrm{kHz}$, le pendant de l'approche intégrale de $\overrightarrow{\operatorname{rot}}(\vec{B})$ (voir 6)serait résolu, mais pour le terme . Ce $\mu_{0} \epsilon_{0}(d \vec{E} / d t)$ terme dit de courant de déplacement a la particularité de ne pas nécessiter de support matériel pour ce développer. Dans ces conditions, une approche locale, fondée sur la mesure de la composante tangentielle de $\vec{B}$ le long d'un circuit pseudo élémentaire immatériel devient envisageable. Telle manipulation serait conceptuellement de même nature que celle décrite en partie 7, autour de l'opérateur divergence, mais cette fois pour le rotationel. En particulier, elle fournirait une aide concrète à la compréhension de la démonstration de la formule de Stokes.

Cette préoccupation appelle la suivante autour de $\overrightarrow{\operatorname{rot}}(\vec{E})$. La discussion amorcée au $\mathbf{4 . 1}$ en donne la contrainte : l'actuel niveau de bruit de la sonde $\mathbf{E}$ doit être réduit d'environ un facteur 100 pour rendre l'expérience faisable. La question préalable est d'en évaluer la faisabilité. Les travaux de T. Clark [10], relatifs à un procédé très faible bruit de mesure de potentiel la montre. Par ailleurs, le développement actuel de notre sonde E n'a pas intégré de contraintes drastiques sur le bruit, néanmoins nous en connaissons les tenants et donc nous sommes en mesure d'estimer la marge de manœuvre à notre disposition pour aller vers de très faible niveaux de bruits. Une diminution de plus d'un facteur $100 \mathrm{du}$ niveau de bruit en champ E équivalent en entrée est clairement envisageable. La manipulation concrète serait alors de mesurer localement le champ électrique à l'intérieur des bobines de Helmoltz utilisées 
dans les expériences décrites au paragraphe 4, au lieu de mesurer sa circulation le long d'un circuit conducteur.

Pour clore ce premier volet de perspectives, l'aspect local de l'opération $\operatorname{div}(\vec{E})$ est également à couvrir. La solution est peut être plus simple qu'il n'y paraît de prime abord : pourquoi ne pas utiliser un simple pendule miniature constitué d'une bille suspendue par un fil isolant et oscillant à quelques $\mathrm{Hz}$ ? Chargé par intermittence par une sonde à un potentiel donné (bille métallique), ou par frottement et action triboélectrique sur une bille isolante la bande passante de notre sonde permet en effet de repérer la valeur du champ statique accompagnant l'objet en mouvement ! Sans aucun doute, cette expérience d'une simplicité étonnante nous accompagnera lors de la manifestation CETSIS 2010, car depuis la soumission de cette proposition, nous avons réalisé de tels pendules, à bille de verre ou de laiton, que nous avons présentés à l'édition de novembre 2009 de la fête de la science à Caen. Le succès auprès des visiteurs, notamment des plus jeunes d'entre eux était très clair. Par réduction du diamètre de la bille, et compte tenu de la facilité de la charger à fort potentiel par simple frottement ou par machine de Van de Graaf, il est plus que probable que les expériences analogues à celles présentées ici pour $\operatorname{div}(\vec{B})$ aient leur exact pendant, mais pour le champ $\vec{E}$.

\subsubsection{Thèmes connexes aux équations de Maxwell}

Un thème directement en aval des équations de Maxwell est celui de la détection électromagnétique et de leurs nombreuses applications, notamment en contrôle non destructif. Nous suggérons qu'au delà des premières utilisations en TP de physique de capteurs capacitifs à électrodes métalliques, comme utilisés dans la sonde E (partie 5), et au delà de l'utilisation de capteurs inductif à simple boucle de capture (partie 4), ces capteurs puisse être repris, mais au travers de la grille du conditionnement électronique, aux niveaux L3 ou M1 des formations en SPI. Tel chaînage permettrait, par le ressort de la récurrence de revisiter, en les approfondissant, ces phénomènes fondamentaux dont l'on sait pertinemment bien que la tendance naturelle est de les oublier rapidement. Le développement de maquettes dédiées, sous forme éclatée, nous apparaît en effet plutôt d'approche facile, car les conditionneurs mis en œuvre ont été conçus en suivant l'essentiel des canons de l'électronique linéaire, et au moyens d'outils de CAO dédié mais gratuits et libres de droits d'utilisation.

Un thème plus encore en aval, est associé aux applications de contrôle non destructif de matériaux, soit suivant la technique traditionnelle de courants de Foucault, soit suivant des techniques émergentes fondées sur l'électrostatique [11]. Nous nous interrogeons sur la pertinence de concevoir des bancs simplifiés dédiés à des TP introductifs à ces applications, non seulement importantes au niveau industriel, mais surtout en lien direct avec les phénomènes fondamentaux de l'électromagnétisme. D'un strict point de vue pédagogique, ce type de manipulation utilisant les savoirs fondamentaux, (objets de ces présents travaux), les savoirs de base de l'électronique et des capteurs, introduirait ceux de l'acquisition et du traitement de données, suivant le moteur récurrence - approfondissement invoqué tout au long de notre article. Fig. 11 illustre notre propos : une image de basse résolution a été obtenue par quelque 25 scans parallèles, à vitesse constante sur une plaque bi ou tri couche de plexiglas, elle montre que des charges persistentes sont présentes, disposées visiblement de façon liées aux interfaces de l'échantillon. Au final, et c'est une question que nous posons en fin de cet article à la communauté du CETSIS, y aurait - il intérêt pédagogique à développer ce genre de système d'acquisition de données avec une assez basse résolution, disons 50x50 pixels, permettant aux étudiants de faire pas à pas le trajet de la génération d'information utiles au niveaux industriels et sociétaux, à partir des phénomènes fondamentaux?
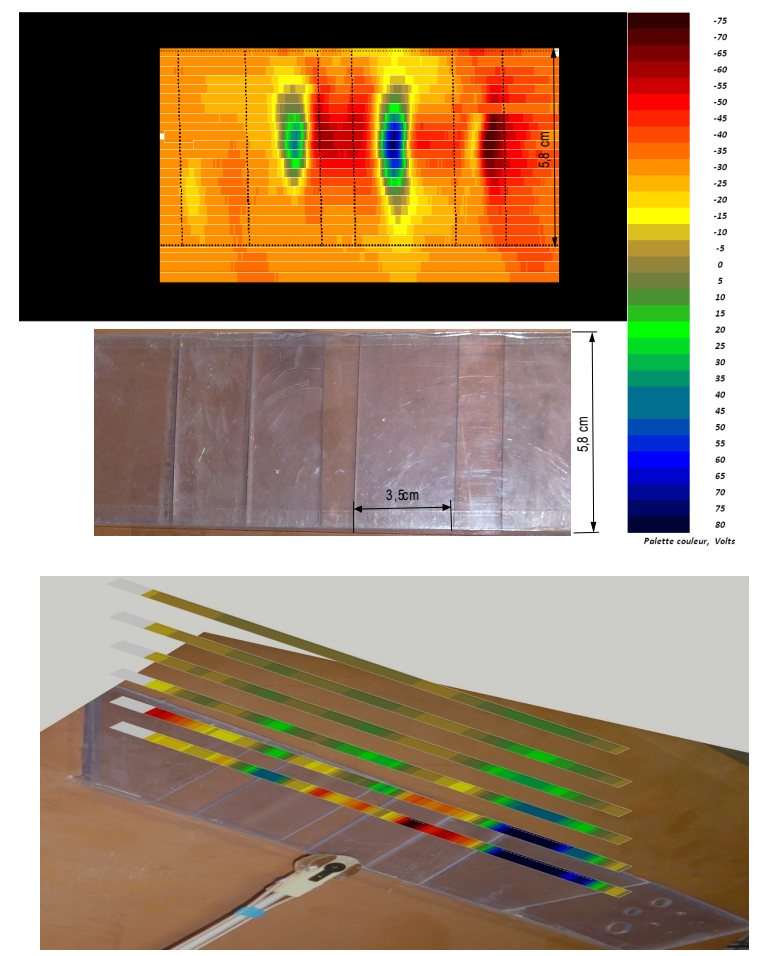

Fig. 11 : Images obtenues par scans à vitesse constante, et possibilités d'imagerie électrostatique simplifée. La palette de couleur va de $-75 \mathrm{~V} \grave{a}+75 \mathrm{~V}$.

\section{CONCLUSIONS}

Cette longue contribution, qui résulte d'une plus longue démarche encore, nous a permis d'aborder la problématique de l'innovation, dans ses dimensions pédagogiques, en lien avec la recherche, en rupture avec les pratiques traditionnelles qui inhibent souvent l'interdisciplinarité par de contre productifs cloisonnements disciplinaires. Une autre dimension est également pré- 
sente, mais non mise en avant : il s'agit de celle de la valorisation industrielle de matériels pédagogiques. Cette dernière dimension, qui n'est pas des plus simple, a fait l'objet de notre contribution [3]. L'argumentaire de notre contribution part d'un constat, celui du manque d'une série de travaux pratiques dédiés et unifiés autour des équations de Maxwell. Il nous a conduit à l'élaboration d'un cahier des charges intégrant explicitement des fonctionnalités requises en lien fort avec une approche pédagogique, le tout visant une meilleure appropriation intellectuelle de ces équations, notamment au travers de complémentarités entre les approches expérimentales, les approches numériques et analytiques. Un trait marquant de nos propositions porte sur le choix des expériences, qui doivent correspondre au mieux à des configurations académiques habituellement traitées uniquement au tableau noir, en cours magistral ou en séance de travaux dirigés. Un autre trait marquant, est le lien avec les mathématiques de l'ingénieur, puisque les grandeurs manipulées au cours de ces expériences peuvent apparaître comme corps d'épreuve pour les opérations de base de l'algèbre vectorielle de base, au travers de réalisations explicites d'opération vectorielles (projection, gradient, divergence, rotationel, intégrales curvilignes, multiples, etc). Un dernier trait important est celui de l'estimation de grandeurs physiques intégrales par échantillonnage spatial, dès les premières expériences, avec visualisation et replacement des grandeurs au moyen d'une technique d'avatar virtuels représentant, à l'écran, le matériel présent sur la table de manipulation et les résultats obtenus, issus de mesure, simulation numériques et/ou calculs analytiques. Des matériels dédiés, capteurs et supports d'expériences innovants sont utilisés pour mettre en œuvre notre démarche pédagogique. Ces innovations, plutôt de type «rupture $»$, justifient le peu de référence à des travaux autres que les nôtres.

Notre approche pourra apparaître complexe, car nous visons des acquisitions simultanées mais progressive d'outils et de méthodes utilisées traditionnellement en sciences pour l'ingénieur et en sciences physiques. Dans les approches habituelles, les difficultés sont abordées avec en général un fort cloisonnement disciplinaire, et de façon plutôt séquentielle. Elles aboutissent souvent à une faible cohérence des manipulations entre elles, et parfois un très grand décalage entre les enseignements formels qui devraient y être connectés. A contrario, nous proposons liens, récurrence et approfondissement successifs, de séance à séance, de semestre à semestre. Il est en effet entendu que l'apprentissage de l'électromagnétisme porte en général sur plusieurs semestres. Notre approche se veut permettre un grand lien entre champs disciplinaires autour d'un thème fédérateur fondamental qui est celui des équations de Maxwell. Il est probable qu'une approche semblable puisse également se mettre en œuvre dans d'autres champs disciplinaires faisant intervenir d'autres grandeurs scalaires et vectorielles.

\section{Remerciements :}

Nous tenons à remercier les acteurs des dispositifs institutionnels pour leur soutien : l'Université de Caen Basse Normandie (UCBN), le Conseil Régional de Basse Normandie (CRBN), Normandie Incubation, OSEO-Anvar, l'ANRT, SYNERGIA. Une attention particulière va à Mr. Jean François Le Bourhis, IA-IPR de Sciences Physiques pour son soutien constant.

\section{Bibliographie}

[1] Didier Robbes, «Sensor for non contact electric field measurements », European Patent Application EP2040086, demandeur Inphynix, $N^{\circ}$ 07291123.3, date : 21.09.2007.

[2] Remarque d'un participant anonyme, Atelier animé par D. Robbes, "Expériences autour des Equations de Maxwell », Congrès national des Professeurs de Physique et de Chimie du 27 au 30 octobre 2008 à Rouen

[3] Didier Robbes, Frédéric Dijoux, «Synergie enseignement-valorisation-recherche », accepté à CETSIS 2009.

[4] Maria Timofeeva, Gilles Allègre, Sthéphane Flament et Robbes Didier, « Des lois de l'induction aux théorèmes de Thevenin et de Norton », accepté à CETSIS 2009.

[5] Didier Robbes, «physical quantity measuring unit and sensor for non contact electric or magnetic field measurement », PCT Patent Application WO2009036989, demandeur Inphynix, $N^{\circ} 08802408.8$, date 19.09.2008.

[6] Didier Robbes, «Physical Quantity Measuring Unit and Device for Measuring a Voltage and an Electric Field »European Patent Application No. 09290213.9, date 20.3.2009

[7] Logiciel Google «SketchUp », pour créer, modifier et partager des modèles 3D. Version gratuite juste limitée en format de fichiers de sortie.

[8] David Meeker, «Finite Element Method Magnetics, FEMM » $A$ Windows finite element solver for $2 D$ and axisymmetric magnetic, electrostatic, heat flow, and current flow problems with graphical pre- and post-processors

[9] Robbes Didier, Pichon Mathieu, "Magnétostatique, algèbre vectorielle: une série de T.P. mise en place en DEUG STPI à Caen", J3eA, Journal sur l'enseignement des sciences et technologies de l'information et des systèmes, Volume 4, Hors-Série 2, 30 (2005)

[10] Clark Terence, Prance Robert, Harland Christopher, «EP 1451595 : Electrodynamic sensors and applications thereof », European Patent Application $N^{\circ} 02783308.6$, 09-122002

[11]C A Rezende, R F Gouveia, M A da Silva and F Galembeck, " Detection of charge distributions in insulator surfaces », J. Phys.: Condens. Matter 21 (2009) 263002 (19pp) 